Peer Reviewed Paper openaccess

\title{
Ultraviolet-visible/near infrared spectroscopy and hyperspectral imaging to study the different types of raw cotton
}

\author{
Mohammad Al Ktash,, ${ }^{\mathrm{a}, \mathrm{b}}$ Otto Hauler, ${ }^{\mathrm{a}}$ Edwin Ostertaga and Marc Brecht ${ }^{\mathrm{a}, \mathrm{b} *}$ \\ aLehr- und Forschungszentrum Process Analysis and Technology (PA\&T) der Hochschule Reutlingen, Alteburgstraße 150,72762 Reutlingen, \\ Germany \\ bIPTC and LISA+ center, University of Eberhard Karls Tübingen, Auf der Morgenstelle 18, 72076 Tübingen, Germany \\ Contacts \\ Mohammad Al Ktash: mohammad.alktash@reutlingen-university.de; \\ https://orcid.org/0000-0002-6713-5669 \\ Otto Hauler: otto.hauler@reutlingen-university.de; \\ https://orcid.org/0000-0002-8858-544X \\ Edwin Ostertag: edwin.ostertag@reutlingen-university.de; \\ https://orcid.org/0000-0003-0492-0544 \\ Marc Brecht: marc.brecht@reutlingen-university.de; \\ https://orcid.org/0000-0001-5537-1448
}

Different types of raw cotton were investigated by a commercial ultraviolet-visible/near infrared (UV-Vis/NIR) spectrometer (210-2200nm) as well as on a home-built setup for NIR hyperspectral imaging (NIR-HSI) in the range 1100-2200nm. UV-Vis/NIR reflection spectroscopy reveals the dominant role proteins, hydrocarbons and hydroxyl groups play in the structure of cotton. NIR-HSI shows a similar result. Experimentally obtained data in combination with principal component analysis (PCA) provides a general differentiation of different cotton types. For UV-Vis/NIR spectroscopy, the first two principal components (PC) represent $82 \%$ and $78 \%$ of the total data variance for the UV-Vis and NIR regions, respectively. Whereas, for NIR-HSI, due to the large amount of data acquired, two methodologies for data processing were applied in low and high lateral resolution. In the first method, the average of the spectra from one sample was calculated and in the second method the spectra of each pixel were used. Both methods are able to explain $\geq 90 \%$ of total variance by the first two PCs. The results show that it is possible to distinguish between different cotton types based on a few selected wavelength ranges. The combination of HSI and multivariate data analysis has a strong potential in industrial applications due to its short acquisition time and low-cost development. This study opens a novel possibility for a further development of this technique towards real large-scale processes.

Keywords: hyperspectral imaging, cotton, pushbroom imaging, NIR spectroscopy, UV-Vis spectroscopy, principal component analysis

\section{Introduction}

Hyperspectral imaging (HSI) is an imaging technology that combines spatial information with spectroscopy. It is a fast and non-destructive method, which has evolved into a powerful analysis tool for product inspection. Thereby, spatial images with very detailed spectral information for each pixel of an object are collected simultaneously. ${ }^{1-3}$ In the past, spectroscopic applications as well as HSI in the ultraviolet-visible (UV-Vis) and near infrared (NIR)
Correspondence

Marc Brecht (marc.brecht@reutlingen-university.de)

Received: 6 November 2020

Revised: 13 December 2020

Accepted: 14 December 2020

Publication: 16 December 2020

doi: 10.1255/jsi.2020.a18

ISSN: 2040-4565

\author{
Citation \\ M. Al Ktash, O. Hauler, E. Ostertag and M. Brecht, "Ultraviolet-visible/ \\ near infrared spectroscopy and hyperspectral imaging to study the differ- \\ ent types of raw cotton", J. Spectral Imaging 9, a18 (2020). \\ https://doi.org/10.1255/jsi.2020.a18 \\ (c) 2020 The Authors \\ This licence permits you to use, share, copy and redistribute the paper in \\ any medium or any format provided that a full citation to the original \\ paper in this journal is given, the use is not for commercial purposes and \\ the paper is not changed in any way.


range are more frequently found in the textile research and in industrial applications. ${ }^{4}$ In textile research, cotton plays a dominant role among textiles, since cotton is the most important naturally occurring raw material for the production of fabrics. 5,6 More than 34 million hectares of land are used to grow cotton, and around 100 million households worldwide are engaged in cotton production. ${ }^{7}$ Cotton is considered as a key resource in the textile industry and accounts for about $30 \%$ of all fibres used in this sector. ${ }^{8}$ In recent years, the increase in quality and processing requirements has led to the introduction of modern techniques for processing and quality control. ${ }^{9-12}$ Nevertheless, distinguishing between different cotton species is still a demanding task.

Several detection methods have been developed and applied to identify and classify different cotton varieties. ${ }^{6,13}$ Most of them are off-line techniques such as thermogravimetric analysis and optical spectroscopy. ${ }^{14-16}$ Only little information is expected in the visible range, since most raw cotton and residuals are reflective (or transparent). ${ }^{17,18}$ Valuable information can be expected in the NIR region from characteristic molecular vibration, e.g. $\mathrm{CH}_{n}$ and $\mathrm{OH}$ groups of cotton which are omnipresent. ${ }^{19}$ Unfortunately, the overall sensitivity for small variations of the sample as well as for small amounts of contaminations in the NIR range is low and they are hard to detect. ${ }^{20,21}$ Therefore, numerous studies in the NIR region used a combination of spectroscopy and chemometric modelling. ${ }^{19,22-28}$

With a NIR-HSI system, a complete optical spectrum with innumerable spectra are collected at all image pixels. This is in contrast to multispectral systems, such as red-green-blue (RGB) cameras, where only a limited number of wavebands are collected. ${ }^{29,30}$

Most HSI applications have been focused on remote sensing systems, such as satellites or aircraft, to gather information for agricultural, geological inspections and military purposes. Nowadays, HSI is evolving into a standard for inline and online inspection in process analytics and quality control. Prominent technical applications can be found in quality control for medicine, food and agricultural products. ${ }^{29,31,32}$

In industrial applications, an HSI system is based on a combination of a pushbroom scanner and a conveyor belt. The pushbroom scanner is fixed over the conveyor belt as shown in Figure 1. Such inspection systems require a minimum of sample preparation and are able to scan several samples swiftly with high spectral resolution. ${ }^{33}$ Here, the pushbroom scanner captures the complete spectral information line by line. The data is collected with the camera placed perpendicular to the conveyor belt. As the conveyor belt moves, images are continuously captured by the pushbroom scanner, resulting in a three-dimensional (3D) data matrix with dimensions $x, y$ and $\lambda$ and is often referred to as hypercube. ${ }^{34}$

For cotton research, HSI was used in the UV-Vis range to detect foreign matter with differentiation and classification of lint in cotton samples. ${ }^{13}$ The results showed great potential for the use of an HSI system for the classification of foreign matter. 4,11,12

In this study, we used optical reflection spectroscopy in the UV-Vis/NIR range as well as HSI in the NIR range for the differentiation of cotton sample sets. For both methods, a chemometric model was developed that is based on principal component analysis (PCA). Using this model, we were able to distinguish between the different cotton types of our sample sets.

\section{Materials and methods UV-Vis/NIR spectroscopy}

Reflectance spectra of the samples were recorded in the range from $210 \mathrm{~nm}$ to $2200 \mathrm{~nm}$ using a UV-Vis/NIR spectrometer (Lambda 1050, PerkinElmer Ltd). It was used to compare the data from the NIR-HSI and validate to another device. The UV-Vis/NIR spectrometer was equipped with an Ulbricht sphere covered by polytetrafluoroethylene (PTFE) to acquire data in diffusion reflection mode with two detectors: one is an indium gallium arsenide (InGaAs) detector and the other a photomultiplier inside the sphere. The samples were placed on this rear of the sphere, and a diffuse scattering PTFE as a white reference disc was placed behind the sample. The complete measuring aperture area is approximately $4.9 \mathrm{~cm}^{2}$. From every cotton sample disc, a spectrum was acquired on each side. In total, three discs were measured for each sample and, thus, for each cotton sample disc, six spectra were recorded.

\section{NIR hyperspectral pushbroom online imaging system}

Figure 1A shows the setup of the HSI system used. The hyperspectral system is based on a pushbroom imager connected to a Xencis, Xeva 2.5 - 320 camera equipped with a mercury cadmium telluride ( $\mathrm{HgCdTe})$ detector of $8 \mathrm{~nm}$ spectral resolution with a $30 \mu \mathrm{m}$ slit width. Two halogen lamps illuminate the sample area. PTFE is used as a white reference, while the dark reference is acquired by imaging without any light exposure 


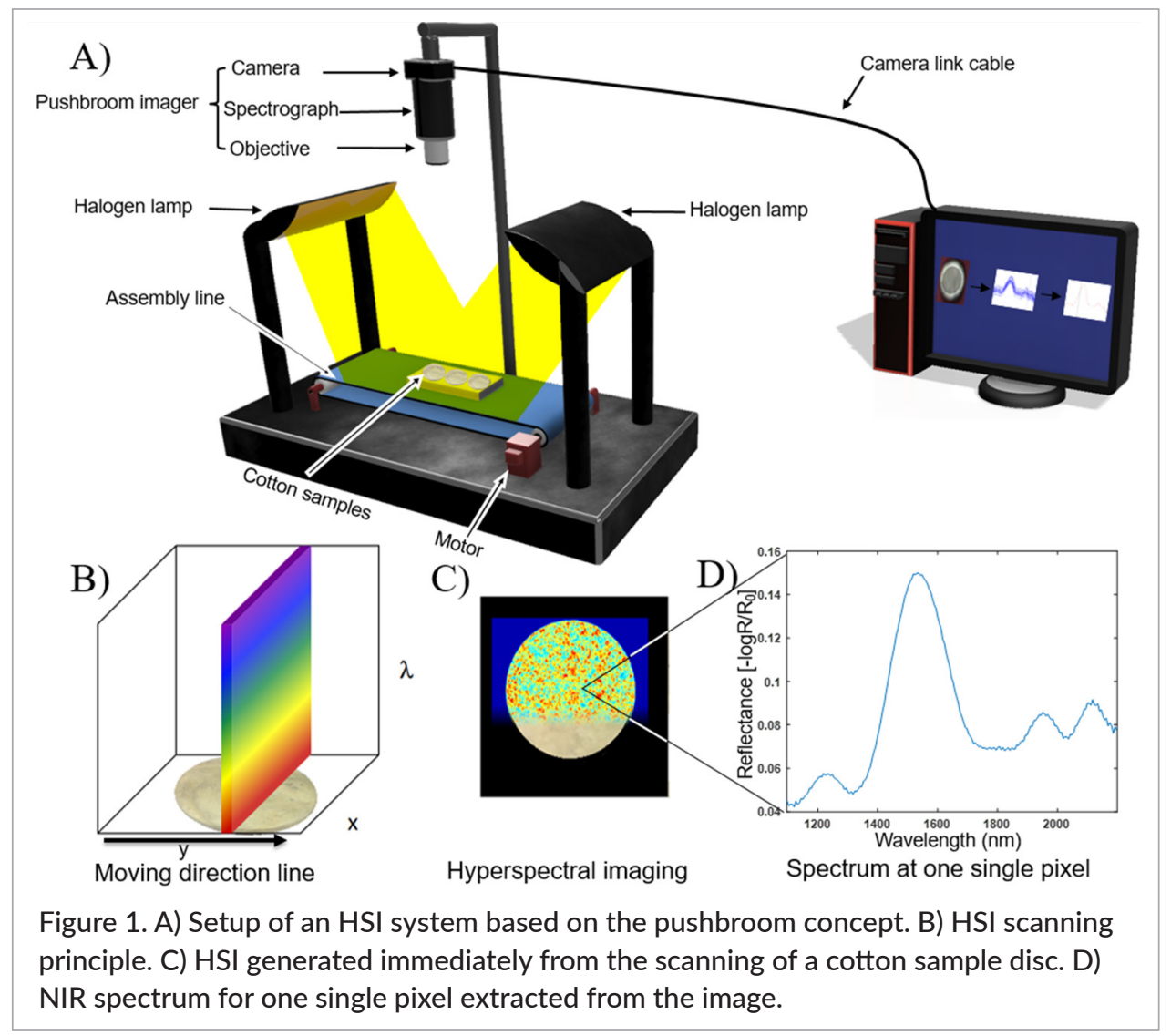

to the sensor. Figure 1(B-D) illustrates the principle and workflow for HSI. Figure 1B shows complete spectroscopic information acquired for each line. Thus, a continuous line-by-line collection of spectral information forms a two-dimensional (2D) image as shown in Figure $1 \mathrm{C}$. It is also possible to extract a single spectrum from a given pixel or point in the $2 \mathrm{D}$ image as shown in Figure 1D.

\section{Samples}

Figure 2 shows five types of raw cotton and one hemp sample which were investigated. The samples are organic raw material cotton (RoB), hemp plant from China ( $H C)$, recycled cotton (RcO), standard raw material cotton (RoSt), recycled organic bright cotton $(\mathrm{RcBH})$ and mechanically cleaned cotton sample (CLN). Three samples of the aforementioned cotton types were collected from the bulk, amounting to $0.75 \mathrm{~g}$ from each sample. The samples were pressed at 10 tons for 2 min by a hydraulic press into a disc shape so that they had the same physical properties. The hydraulic press was cleaned after each sample to reduce the chance of any impurities.



Figure 2. Raw cotton sample discs. 


\section{Data collection and pre-processing of hyperspectral data}

The following two methods for data pre-processing are described, resulting in low and high lateral resolution. Matlab (MATLAB 9.2.0, Mathworks, MA, USA) scripts were written for pre-processing of the hyperspectral data cube.

Figure 3 shows the workflow for calculating the mean spectrum of each sample. The hyperspectral image is collected by moving any cotton sample disc at a constant speed, approximately 50 spectra were collected manually within the indicated area of interest, as shown in Figure 3A (marked as dashed line) and plotted as shown in Figure 3B. The average of these spectra is calculated and shown in Figure 3C.

Figure 4 shows the workflow for the second preprocessing method. The hyperspectral image is captured by moving the 18 cotton sample discs at a constant speed. To differentiate between signal and background, a distinction is first made between the respective spectral characteristics.

For this purpose, two parallel planes are fitted into each spectral channel, one for the background and one for the samples. The distance between these planes is then selected as the parameter for the spectral difference between the sample and the background. The colour channel with the highest value is used as a mask for all other colour channels. Half of this difference is set as the threshold value. All lateral points of the colour channel whose intensity value is above this threshold value are classified as background and removed. This clipping mask is applied to the entire hyperspectral data cube. The remaining data corresponds to the spectral contributions from the samples. These are converted from the 3D hyperspectral data set into a 2D format by joining the lateral points of the $X$ and $Y$ dimensions. This creates a matrix in which each row corresponds to a pixel with a complete spectrum. This matrix is used as input for the PCA.
Figure 4A shows the image obtained through the hyperspectral camera. The colour channel with the highest differential value is displayed in Figure 4B. Figure 4C shows a single colour channel of the cotton sample discs' hyperspectral data cube after removing all lateral components associated with the background, the removal of outliers like dead pixels or cosmic events, and the application of a PCA filter, which removes all contributions of higher PCA components. The PCA filter works as follows: the first three of the resulting PCs explain about $88 \%$ of the variance. The $4^{\text {th }}$ and higher components, while contributing less than $7 \%$ to the overall variance, contain mainly noise and were therefore discarded for further analysis. The remaining $5 \%$ of the total variance is found within the residuals, and does not contribute significant information. Figure 4D shows an image, where the RGB value corresponds to scores of the first $(R)$, second $(G)$ and third (B) components.

In the next step, all score values that are $90 \%$ similar to another score in all the main components considered are removed from the data set. From the remaining score values a reduced data set with the load values of the considered main components was generated. The reduced data set is then converted back into a 3D hyperspectral data cube by separating the combined lateral information. Figure 4C shows the reduced data as lateral information for one spectral channel. The principal component analysis of this data again shows a significant grouping of the different types of cotton. In the end, approximately 120,000 spectra remain from the initially obtained 1.7 million spectra.

\section{Data handling and software}

The UV-Vis/NIR spectra are recorded with the Lambda 1050 UV WinLab software from PerkinElmer. The NIR hyperspectral pushbroom images are analysed by the Evince 2.7.9 software from Prediktera. PLS Toolbox 8.5.1 (Eigenvector Research, Inc., USA) is used for the data

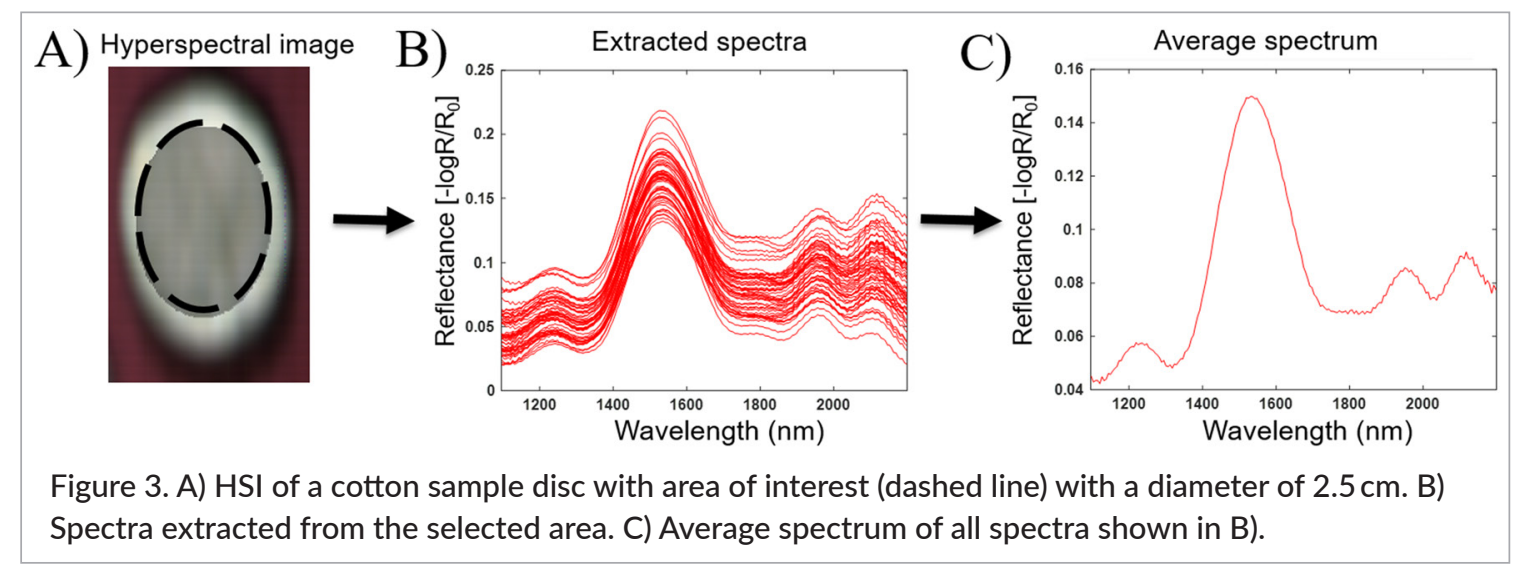




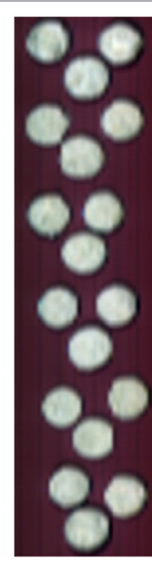

A

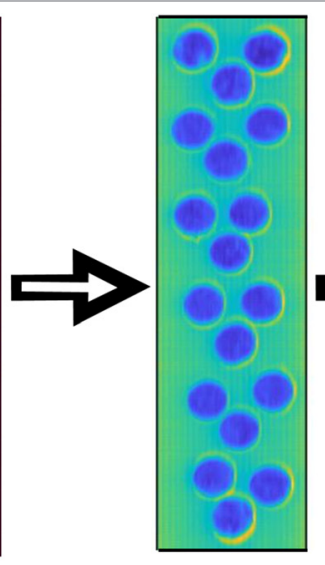

B

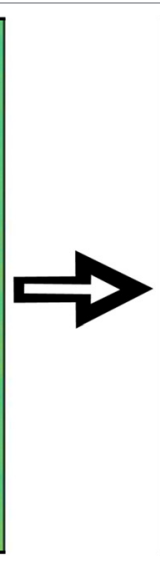

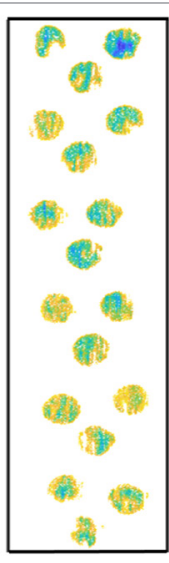

C

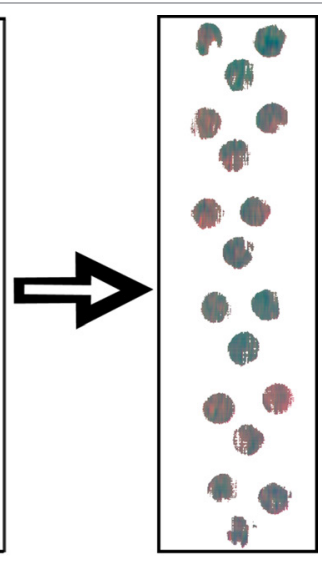

D

Figure 4. A) Hyperspectral raw imaging of 18 cotton sample discs with a diameter of $3.1 \mathrm{~cm}$. B) Image of the colour channel with the highest variance between cotton disks and background. C) Images after subtraction of the background, removal of outliers and application of filters. D) Image of RGB value corresponding to scores of the first (R), second (G) and third (B) components.

processing and analysis. Lighting conditions may vary between the samples and even within the samples across the scan line. A common way to calculate this effect is to convert measured raw spectra to reflectance spectra by the following formula: ${ }^{29,35}$

$$
\text { Reflectance }=-\log R / R_{0} \frac{I_{\text {sample }}-I_{\text {dark }}}{I_{\text {reference }}-I_{\text {dark }}}
$$

where $R$ and $R_{0}$ represents the transmitted and incident intensity. $I_{\text {sample }}$ is the intensity of the original image data, $I_{\text {dark }}$ is the intensity of the dark current image data and $I_{\text {reference }}$ is the intensity of the white reflectance image. Pre-processing of the mean centre, smoothing (SavitzkyGolay) with filter width 15 and polynomial order one, and generalised least squares (GLS) are applied to the data. GLS is used to achieve an efficiency by transforming the variance covariance matrix into a homoscedastic one. ${ }^{36}$ It works as a filter that calculates the differences between the samples. The differences are considered as interference or clutter and GLS aims to reduce these interferences. $^{36-38}$

\section{Results and discussion UV-Vis/NIR spectroscopy}

Figure 5A shows UV-Vis/NIR spectra (210-2200 nm) from all samples. Six spectra were recorded for each cotton sample type, three on each side. As expected, the spectra show a high similarity. All spectra show the strongest reflectance at $280 \mathrm{~nm}$ which can be attributed to proteins on the samples, see Table $1 .{ }^{17}$ In the visible range from $400 \mathrm{~nm}$ to $750 \mathrm{~nm}$, the spectra do not show any distinct features since most of the raw cotton is reflective. In the NIR region, several spectral features can be observed. Dominant contributions are found at $1500 \mathrm{~nm}$, $1933 \mathrm{~nm}$ and $2100 \mathrm{~nm}$ corresponding to the functional groups $\mathrm{CH}, \mathrm{ROH}$ and $\mathrm{OH}$, respectively.

Due to the high similarity of the spectra, a differentiation of the samples is demanding. As a consequence, PCA is used to further differentiate the samples and was applied for processed spectra.

The processing of spectra is described in the Materials and methods section. Figure 5B shows the scores plot of the first two principal components PC1 and PC2 for the UV-Vis region (210-1100 $\mathrm{nm}$ ). The PCA model explains $70.1 \%$ and $82.3 \%$ of the spectral information with the first two PCs, respectively. The scores plot shows that PC1 and PC2 are sufficient to separate all samples. In this representation, the hemp $(\mathrm{HC})$ sample shows the most distinct separation from the cotton group, as expected. Figure 5C shows the corresponding loadings plot for PC1 and PC2. The most significant differences between those loadings are found in the regions from $210 \mathrm{~nm}$ to $350 \mathrm{~nm}$ and from $450 \mathrm{~nm}$ to $700 \mathrm{~nm}$. In the UV range $(210-350 \mathrm{~nm})$, the strongest influence on PC1 is found at $280 \mathrm{~nm}, 300 \mathrm{~nm}$ and for PC2 at $290 \mathrm{~nm}$. They can be assigned to proteins and amino acids (see Table 1). ${ }^{17}$ The 
contributions in the visible range $(450-700 \mathrm{~nm})$ show a maximum/minimum at $680 \mathrm{~nm}$, it can be assigned to the colour of the RcO samples (see also the inset in Figure 5A).

Figure 5D shows the scores plot of the first two principal components PC1 and PC2 for the NIR region (1100-2200 nm). The PCA model explains $63.5 \%$ and $78.0 \%$ of the spectral information with the first two PCs, respectively. The scores plot shows that the first two PCs are sufficient to separate all samples clearly from one another in the NIR range. In the scores plot, the hemp $(\mathrm{HC})$ and $\mathrm{CLN}$ sample shows the most distinct separation from the cotton group.

Figure 5E shows the loadings plot for PC1 and PC2. The most significant differences between those loadings are found in the regions of $1100-1200 \mathrm{~nm}, 1350-1500 \mathrm{~nm}$, $1600-1700 \mathrm{~nm}$ and $1850-2100 \mathrm{~nm}$. In the NIR region (1100-2200 nm), several spectral features are variable which are assigned to the hydrocarbons and hydroxides oscillation (see Table 1). ${ }^{17}$

With UV-Vis/NIR, a separation of the analysed cotton sample discs has been successfully demonstrated. However, the large deviations between PC1 and PC2 are mainly found in the UV-Vis and NIR regions. Therefore, the application of an online method for characterisation is the most suitable for these spectral regions.

\section{NIR hyperspectral imaging}

Two data processing techniques were applied to the NIR hyperspectral images to calculate PCA models. As before, three samples of each raw fibre were analysed. The setup for HSI as well as for determination of the spectra from the hyperspectral data matrix is described in the Materials and methods section.

In the first method, the mean value of the spectra was calculated for each cotton sample disc. A total of six spectra are determined from the HSI data for each cotton sample type.

Figure 6A shows hyperspectral NIR spectra in the range from $1100 \mathrm{~nm}$ to $2200 \mathrm{~nm}$. The most dominant contributions are observed around $1525 \mathrm{~nm}$, which can be attributed to the presence of $\mathrm{OH}$ groups. Four weaker peaks are observed around $1340 \mathrm{~nm}, 1790 \mathrm{~nm}, 1955 \mathrm{~nm}$ and $2117 \mathrm{~nm}$, their assignment is given in Table $2{ }^{20}$

The PCA of these spectra explains $93.7 \%, 97.0 \%$ or $98.3 \%$ of the spectral information with the first two, three or four PCs, respectively. Figure 6B shows the results for the first three PCs. In the scores plot it can be seen that the first three PCs are sufficient to separate all samples clearly from one another.

Figure 6C shows the loadings plot for the first three PCs. In the range from $1340 \mathrm{~nm}$ to $1663 \mathrm{~nm}$ the reflectance around $1508 \mathrm{~nm}$ can be assigned to the presence of $\mathrm{ROH}$ (see Table 2). In reflectance in the range from $1789 \mathrm{~nm}$ to $2100 \mathrm{~nm}, 1973 \mathrm{~nm}$ can be assigned to the $\mathrm{OH}$ group. The contribution at approximately $2270 \mathrm{~nm}$ is due to $\mathrm{CH}^{39,40}$

In the second method, several thousand spectra from every cotton sample disc were used to calculate the PCA model. The pre-processing and workflow of the spectra from the hyperspectral data matrix is described in the Materials and methods section. Figure 7A presents examples of hyperspectral NIR spectra from a single pixel of each of the six cotton types in the range $1100-2200 \mathrm{~nm}$.

The PCA of these spectra explains $86.0 \%, 88.2 \%$ or $89.0 \%$ of the spectral information with the first two, three or four PCs, respectively. Figure 7B shows the results for the first three PCs, the first three PCs are sufficient to separate all samples from one another. A clear separation is observed for RoB and CLN, while the $\mathrm{HC}$, RoSt, RcO and RcBH are slightly overlapping. Nevertheless, these samples can be separated only if

Table 1. UV-Vis/NIR reflectance maxima.

\begin{tabular}{|l|l|l|}
\hline Reflectance $(\mathrm{nm})$ & Functional groups & References \\
\hline $280 \mathrm{~nm}$ & Protein and amino acids & 17 \\
\hline $1210 \mathrm{~nm}$ & $\mathrm{CH}_{2}, \mathrm{CH}$ & 39,40 \\
\hline $1375 \mathrm{~nm}$ & $\mathrm{CH}_{3}$ & 39,40 \\
\hline $1480-1580 \mathrm{~nm}$ & $\mathrm{H}_{2} \mathrm{O}, \mathrm{ROH}, \mathrm{CH}$ & 39,40 \\
\hline $1775 \mathrm{~nm}$ & $\mathrm{CH}_{3}, \mathrm{CH}$ & 39,40 \\
\hline $1933 \mathrm{~nm}$ & $\mathrm{H}_{2} \mathrm{O}, \mathrm{ROH}$ & 39,40 \\
\hline $2100 \mathrm{~nm}$ & $\mathrm{ROH}$ & 39,40 \\
\hline $2275 \mathrm{~nm}$ & $\mathrm{CH}_{3}, \mathrm{CH}_{2}, \mathrm{CH}$ & 39,40 \\
\hline $2340 \mathrm{~nm}$ & $\mathrm{CH}_{3}, \mathrm{CH}_{2}, \mathrm{CH}$ & 39,40 \\
\hline
\end{tabular}






any one pair, e.g. HC and RoSt, is included in a separate model (data not shown). Figure 7C shows the loadings plot of the first three PCs. Overall, the loadings are comparable with the loadings shown in Figure 6C, except a change of the sign. In the range from $1350 \mathrm{~nm}$ to $1700 \mathrm{~nm}$, the reflectance around $1550 \mathrm{~nm}$ can be assigned to the presence of $\mathrm{ROH}$ (see Table 2). The reflectance in the range from $1800 \mathrm{~nm}$ to $1990 \mathrm{~nm}$ can be assigned to $\mathrm{OH}$ groups. The signal around $2302 \mathrm{~nm}$ is due to $\mathrm{CH}^{39,40}$ 




Figure 6. A) Spectra recorded by HSI of cotton sample discs including one $\mathrm{HC}$ sample in the NIR range from $1100 \mathrm{~nm}$ to $2200 \mathrm{~nm}$. Upper right: image of a cotton sample disc where the region of integration for determining the average spectra for each sample is indicated by a black circle with a diameter of $2.5 \mathrm{~cm}$. B) Scores plot for the processed spectra in NIR-HSI. The 2D projection of the $70 \%$ confidence ellipse of the data collected from each type of cotton is included to facilitate visualisation of the obtained results. C) Loadings plot for the NIR-HSI.

The first three PCs explain a significant amount of the NIR hyperspectral data for both pre-processing methods. Calculating the PCA model at each pixel or deriving it from the mean spectra does not significantly change the data behaviour of the model (Figure 6 and Figure
Table 2. HSI-NIR reflectance maxima.

\begin{tabular}{|l|l|l|}
\hline \multicolumn{1}{|c|}{$\begin{array}{c}\text { Reflectance } \\
\text { (nm) }\end{array}$} & Functional groups & References \\
\hline $1240 \mathrm{~nm}$ & $\mathrm{CH}$ & 39,40 \\
\hline $1525 \mathrm{~nm}$ & $\mathrm{ROH}$ & 39,40 \\
\hline $1790 \mathrm{~nm}$ & $\mathrm{CH}_{3}, \mathrm{CH}_{2}$ & 39,40 \\
\hline $1955 \mathrm{~nm}$ & $\mathrm{OH}$ & 39,40 \\
\hline $2117 \mathrm{~nm}$ & $\mathrm{ROH}$ & 39,40 \\
\hline $2342 \mathrm{~nm}$ & $\mathrm{CH}, \mathrm{CH}_{2}, \mathrm{CH}_{3}$ & 39,40 \\
\hline
\end{tabular}

7). The advantage of using average spectra instead of the complete data set is fast data processing. However, this method is limited to recognise or spectrally separate background from the samples automatically. Therefore, a certain time is required to select the samples manually and calculate the average spectra for each cotton sample disc. On the other hand, when applying a filter (see Figure 4) the separation of the sample from the background works automatically, but here the quantity of data hampers a fast processing. The scattering in the scores plot in Figure 7 shows the huge variability of the properties of the samples, theses only become visible if the spectra are taken with HSI. Compared with the scattering where the spectral information is averaged over a larger area (Figure 5 and Figure 6) this is remarkably reduced. The large variability of the score values from the HSI indicates a change of the sample's properties on the scale of the resolution actually achieved. For the $\mathrm{HSI}$ setup this is about $13 \mu \mathrm{m}$. The high lateral resolution achieved here shows that sample properties on this scale vary and are therefore relevant, as new insights into the heterogeneity of fibre samples can be gained. As a consequence, the data show the high potential for HSI which is beyond the differentiation of fibre types.

In the next step, a filter is required that combines the advantages of both methods to speed up the data handling. Together with this, a simplified model can be developed that meets the requirements of real online applications.

\section{Conclusions}

UV-Vis/NIR reflection spectroscopy and HSI in combination with PCA is a promising approach for the detection and differentiation of raw cotton types. The most relevant information for the differentiation of cotton types was found in both the UV and NIR range (see Figure 5C). 

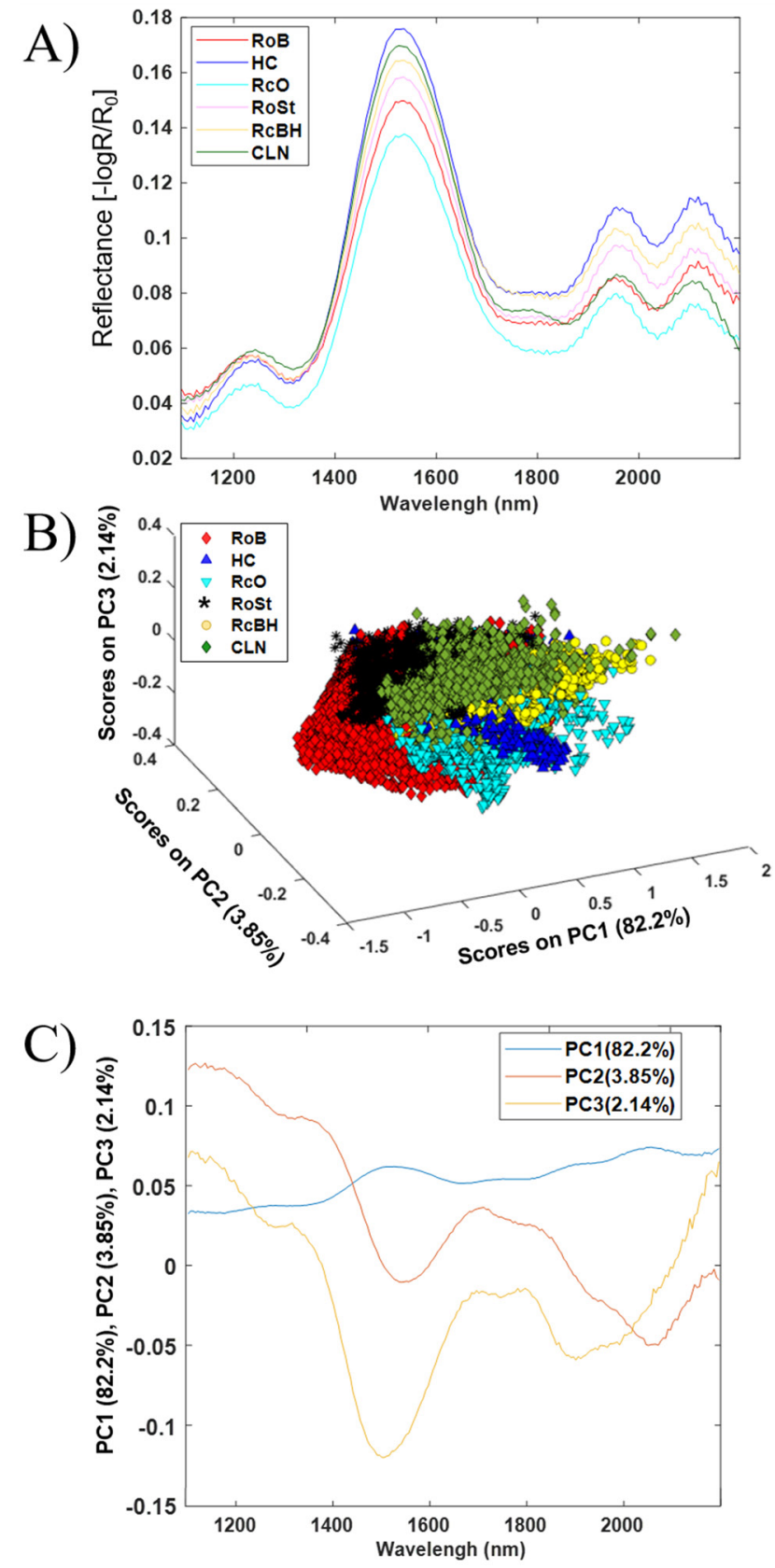

Figure 7. HSI of cotton sample discs including one $\mathrm{HC}$ sample in the NIR range from $1100 \mathrm{~nm}$ to $2200 \mathrm{~nm}$. A) Six example spectra recorded at individual pixels. B) Scores plot calculated for the whole data set including several thousand processed spectra. C) Loadings plot for the NIR-HSI.

The results obtained with UV-Vis/NIR spectroscopy revealed that the contribution in the UV can be assigned to the presence of protein at $280 \mathrm{~nm}$. The most dominant contribution to absorbance in the NIR range can be assigned to $\mathrm{CH}_{3}$ for the most prominent band at $1775 \mathrm{~nm}$ and to $\mathrm{ROH}$ vibrations at $1500 \mathrm{~nm}$. The spectral data were analysed with PCA in order to achieve a differentiation of different cotton types. The PCA model was able to classify all types with the first two PCs explaining the maximum variance of the data.

NIR-HSI results reveal the most dominant absorbance assigned to $\mathrm{CH}_{3}$ and $\mathrm{ROH}$ at $2270 \mathrm{~nm}$ and $1525 \mathrm{~nm}$, respectively. Two methods were used for processing the large amount of data. Both approaches resulted in a differentiation of all types. The advantages of the rugged online home-built setup is a high spatial/spectral resolution and a rapid data acquisition. With this method, several samples can be measured in a short time and at low cost.

Based on the data shown it is reasonable to develop a simplified chemometric model, which meets the requirements of a real process with industrial standards and precision.

\section{Acknowledgements}

The authors thank Professor Dr Volker Jehle, Reutlingen University, for providing the samples and fruitful discussions; Barbara Boldrini, Tim Bäuerle, Tobias Drieschner and Ashutosh Mukherjee are acknowledged for valuable discussions.

\section{References}

1. G. Lu and B. Fei, "Medical hyperspectral imaging: a review", J Biomed. Opt. 19, 10901 (2014). https://doi. org/10.1117/1.JBO.19.1.010901

2. B. Boldrini, W. Kessler, K. Rebner and R.W. Kessler, "Hyperspectral imaging: a review of best practice, performance and pitfalls for in-line and on-line applications", J. Near Infrared Spectrosc. 20, 483-508 (2012). https://doi.org/10.1255/jnirs.1003

3. J. Stiedl, G. Azemtsop, M.B. Boldrini, S. Green, T. Chassé and K. Rebner, "Characterisation of oxide layers on technical copper based on visible hyperspectral imaging", J. Spectral Imaging 8, a10 (2019). https://doi.org/10.1255/jsi.2019.a10

4. H. Zhang and D. Li, "Applications of computer vision techniques to cotton foreign matter inspection: A review", Comput. Electron. Ag. 109, 59-70 (2014). https://doi.org/10.1016/j.compag.2014.09.004

5. J.P.S. Morais, M. de Freitas Rosa, M. de sá Moreirade Souza Filho, L. Dias Nascimento, D. Magalhãesdo Nascimento and A. Ribeiro Cassales, "Extraction and characterization of nanocellulose structures from raw cotton linter", Carbohyd. Polym. 
91, 229-235 (2013). https://doi.org/10.1016/j.carbpol.2012.08.010

6. L.A. Meyer, The World and U.S. Cotton Outlook for 2019/20. United States Department for Agriculture (2019). https://www.usda.gov/sites/default/files/ documents/Leslie_Meyer.pdf

7. C.E. Pray, J. Huang, R. Hu and S. Rozelle, "Five years of Bt cotton in China-the benefits continue", Plant J. 31, 423-430 (2002). https://doi.org/10.1046/ j.1365-313X.2002.01401.x

8. Sustainable Cotton Ranking 2017, Assessing Company Performance. Pesticide Action Network UK, Solidaridad and WWF International (2017). https:// www.wwf.org.uk/sites/default/files/2017-10/ Sustainable\%20Cotton\%20Ranking\%202017\%20 FA\%20lores\%2020170930.pdf

9. R. Luttrell, G. Fitt, F. Ramalho and E.S. Sugonyaev, "Cotton pest management: Part 1. A worldwide perspective", Ann. Rev. Entomol. 39, 517526 (1994). https://doi.org/10.1146/annurev. en.39.010194.002505

10. J.M. Bradow and G.H. Davidonis, "Quantitation of fiber quality and the cotton production-processing interface: a physiologist's perspective", Cotton Sci. 4, 34-64 (2000). http://intranet.cotton.org/journal/2000-04/1/upload/jcs04-034.pdf

11. Y. Jiang and C. Li, "Detection and discrimination of cotton foreign matter using push-broom based hyperspectral imaging: System design and capability", PLOS One 10, e0121969 (2015). https://doi. org/10.1371/journal.pone.0121969

12. C. Ni, Z. Li, X. Zhang, X. Sun, Y. Huang, L. Zhao, T. Zhu and D. Wang, "Online sorting of the film on cotton based on deep learning and hyperspectral imaging", IEEE Access 8, 93028-93038 (2020). https://doi.org/10.1109/ACCESS.2020.2994913

13. A. Mustafic, Y. Jiang and Li C, "Cotton contamination detection and classification using hyperspectral fluorescence imaging", Textile Res. J. 86, 1574-1584 (2016). https://doi.org/10.1177/0040517515590416

14. A.V. Ghule, R.K. Chen, S.H. Tzing, J. Lo and Y.C. Ling, "Simple and rapid method for evaluating stickiness of cotton using thermogravimetric analysis", Anal. Chim. Acta 502, 251-256 (2004). https://doi. org/10.1016/j.aca.2003.10.021

15. N. Abidi and E. Hequet, "Fourier Transform infrared analysis of cotton contamination", Textile Res. J. 77, 77-84 (2016). https://doi. org/10.1177/0040517507074624
16. J. Was-Gubala and R. Starczak, "Nondestructive identification of dye mixtures in polyester and cotton fibers using Raman spectroscopy and ultraviolet-visible (UV-Vis) microspectrophotometry", Appl. Spectrosc. 69, 296-303 (2015). https://doi. org/10.1366/14-07567

17. A.R. Goldfarb and L.J. Saidel, "Ultraviolet absorption spectra of proteins", Science 114, 156-157 (1951). https://doi.org/10.1126/science.114.2954.156

18. F. Zhou and T. Ding, "Detection of cotton lint trash within the ultraviolet-visible spectral range", Appl. Spectrosc. 64, 936-941 (2010). https://doi. org/10.1366/000370210792081091

19. P. Peets, I. Leito, J. Pelt and S. Vahur, "Identification and classification of textile fibres using ATRFT-IR spectroscopy with chemometric methods", Spectrochim. Acta A 173, 175-181 (2017). https:// doi.org/10.1016/j.saa.2016.09.007

20. J. Rodgers and K. Beck, "NIR characterization and measurement of the cotton content of dyed blend fabrics", Textile Res. J. 79, 675-686 (2009). https:// doi.org/10.1177/0040517508090884

21. C. Chung, M. Lee and E. Choe, "Characterization of cotton fabric scouring by FT-IR ATR spectroscopy", Carbohyd. Polym. 58, 417-420 (2004). https://doi. org/10.1016/j.carbpol.2004.08.005

22. M. Blanco, J. Coello, H. Iturriaga, S. Maspoch and J. Pagès, "Use of near-infrared spectrometry in control analyses of acrylic fibre manufacturing processes", Anal. Chim. Acta 383, 291-298 (1999). https://doi. org/10.1016/S0003-2670(98)00804-6

23. E. Cleve, E. Bach and E. Schollmeyer, "Using chemometric methods and NIR spectrophotometry in the textile industry", Anal. Chim. Acta 420, 163-167 (2000). https://doi.org/10.1016/S00032670(00)00888-6

24. C.A. Fortier, J.E. Rodgers, M.S. Cintrón, X. Cui and J.A. Foulk, "Identification of cotton and cotton trash components by Fourier transform near-infrared spectroscopy", Textile Res. J. 81, 230-238 (2010). https://doi.org/10.1177/0040517510383620

25. C. Ruckebusch, F. Orhan, A. Durand, T. Boubellouta and J.P. Huvenne, "Quantitative analysis of cottonpolyester textile blends from near-infrared spectra", Appl. Spectrosc. 60, 539-544 (2006). https://doi. org/10.1366/000370206777412194

26. Y. Liu, "Recent progress in Fourier transform infrared (FTIR) spectroscopy study of compositional, structural and physical attributes of developmental cotton 
fibers", Materials 6, 299-313 (2013). https://doi. org/10.3390/ma6010299

27. R. Ríos-Reina, D.L. García-González, R.M. Callejón and J.M. Amigo, "NIR spectroscopy and chemometrics for the typification of Spanish wine vinegars with a protected designation of origin", Food Control 89, 108-116 (2018). https://doi.org/10.1016/j.foodcont.2018.01.031

28. S.M. Obeidat, M.S. Khanfar and W.M. Obeidat, "Classification of edible oils and uncovering adulteration of virgin olive oil using FTIR with the aid of chemometrics", Austr. J. Basic Appl. Sci. 3, 2048-2053 (2009).

29. J. Tschannerl, J. Ren, F. Jack, J. Krause, H. Zhao, W. Huang and S. Marshall, "Potential of UV and SWIR hyperspectral imaging for determination of levels of phenolic flavour compounds in peated barley malt", Food Chem. 270, 105-112 (2019). https://doi. org/10.1016/j.foodchem.2018.07.089

30. A. Schneider and H. Feussner, Biomedical Engineering in Gastrointestinal Surgery. Academic Press (2017). https://doi.org/10.1016/B978-0-12-8032305.00001-4

31. P. Colarusso, L.H. Kidder, I.W. Levin, J.C. Fraser, J.F. Arens, E.N. Lewis, "Infrared spectroscopic imaging: from planetary to cellular systems", Appl. Spectrosc. 52(3), 106A-120A (1998). https://doi. org/10.1366/0003702981943545

32. G. Mirschel, O. Daikos, T. Scherzer, C. Steckert, "Near-infrared chemical imaging used for in-line analysis of functional finishes on textiles", Talanta 188, 91-98 (2018). https://doi.org/10.1016/j. talanta.2018.05.050

33. S. Zhu, L. Zhou, P. Gao, Y. Bao, Y. He and L. Feng, "Near-infrared hyperspectral imaging combined with deep learning to identify cotton seed varieties", Molecules 24, 3268 (2019). https://doi.org/10.3390/ molecules 24183268
34. J.M. Amigo and C. Santos, "Preprocessing of hyperspectral and multispectral images", Data Handl. Sci. Technol. 32, 37-53 (2020). https://doi.org/10.1016/ B978-0-444-63977-6.00003-1

35. J.Y. Barnaby, T.D. Huggins, H. Lee, A.M. McClung, S.R.M. Pinson, M. Oh, G.R. Bauchan, L. Tarpley, K. Lee, M.S. Kim and J.D. Edwards, "Vis/NIR hyperspectral imaging distinguishes sub-population, production environment, and physicochemical grain properties in rice", Sci. Rep. 10, 1-13 (2020). https:// doi.org/10.1038/s41598-020-65999-7

36. H. Martens, M. Høy, B.M. Wise, R. Bro and P.B. Brockhoff, "Pre-whitening of data by covarianceweighted pre-processing", J. Chemometr. 17, 153165 (2003). https://doi.org/10.1002/cem.780

37. B.M. Wise, H. Martens, M. Høy, R. Bro and P.B. Brockhoff, "Calibration transfer by generalized least squares", Proceedings of the Seventh Scandinavian Symposium on Chemometrics (SSC7), Copenhagen, Denmark. pp. 19-23 (2001).

38. M.T. Jirata, J.C. Chelule and R. Odhiambo, "Deriving some estimators of panel data regression models with individual effects", Int. J. Sci. Res. 3, 53-59 (2012).

39. Y. Liu, G. Gamble and D. Thibodeaux, "UV/visible/near-infrared reflectance models for the rapid and non-destructive prediction and classification of cotton color and physical indices", J. Trans. ASABE 53, 1341-1348 (2010). https://doi. org/10.13031/2013.32584

40. Y. Liu, C. Delhom, B.T. Campbell and V. Martin, "Application of near infrared spectroscopy in cotton fiber micronaire measurement", Inform. Process. Agric. 3, 30-35 (2016). https://doi.org/10.1016/j. inpa.2016.01.001 